\title{
II. Determinanten des nationalsozialistischen Kriegspotentials: Akademische Theorien und bürokratische Praxis
}

\author{
Analysen der wirtschaftlichen und militärischen Kapazität \\ Deutschlands
}

Einer der Grundgedanken in Donovans „Memorandum of Establishment of Service of Strategic Information“ vom 10.Juni $1941^{1}$ war die Überlegung gewesen, daß der moderne Krieg als "totaler Krieg" die Mobilisierung aller Ressourcen einer Nation notwendig mache und daß deshalb möglichst korrekte Analysen der gesamten Wirtschaftskapazität eines Gegners für sinnvolle strategische Planungen unverzichtbar seien. Gerade mit Blick auf ein "gleichgeschaltetes" und scheinbar entsprechend total mobilisiertes Deutschland mochte dies als allzu offensichtlich erscheinen" ${ }^{2}$ doch reflektierten Donovans Überlegungen auch die grundsätzlich wichtige Erkenntnis, daß die technischen Fortschritte seit dem Ersten Weltkrieg eine immer raschere Entwicklung militärischer Technologien erlaubten und daß das militärische Potential einer Nation so in zunehmendem Maße von einer wachsenden Zahl wirtschaftlicher Faktoren determiniert wurde ${ }^{3}$.

Ein Grundstock von Daten und Informationen für die von Donovan als so wichtig erachteten Wirtschaftsanalysen war in Washington bereits vorhanden. Militärisch relevante Informationen wurden von Army- und Navy-Stellen gesammelt; daneben verfügten eine ganze Reihe von Washingtoner Ministerien und Regierungsbehörden über mehr oder minder ausführliche Datensammlungen zur Wirtschaft anderer Nationen, insbesondere der europäischen Staaten, zumal die USA in der Folge des Ersten Weltkrieges entscheidenden Einfluß auf die Reparationsregelungen genommen hatten. Für die Ausarbeitung aktueller Schätzungen waren diese Materialien nach Donovans Ansicht freilich kaum von Nutzen, solange sie nicht in einer Zentrale systematisch erfaßt wurden ${ }^{4}$. Donovans grundsätzliche Kritik, daß die Arbeit der US-Behörden unter einem Mangel an Koordination leide, mußte um so berechtigter erscheinen, als er 1940 schließlich vor allem deshalb zweimal nach Großbritannien entsandt worden war, weil man in Washington nicht einmal das Potential einer befreundeten Nation hinreichend zuverlässig einzuschätzen wußte.

Diesem empfindlichen Mangel sollten nach Donovans Vorstellungen künftig die in der Economics Division der R\&A-Branch tätigen Wirtschaftswissenschaftler mit ih-

\footnotetext{
${ }^{1}$ Abgedruckt in Troy, Donovan, S. $419 \mathrm{f}$.

2 Das Ausmaß bzw. die Intensität der Mobilmachung der deutschen Wirtschaft wurde anfänglich allgemein überschätzt, dazu unten ausführlicher. Siche zu diesem Themenkomplex Herbst, Der Totale Krieg, besonders S.111ff.; vgl. außerdem Wark, British Military and Economic Intelligence, in: Andrew/Dilks (Hrsg.), The Missing Dimension, S. 78-100.

3 Vgl. dazu Kennedy, The Rise and Fall of the Great Powers, besonders S.275ff. und S.291 ff.

${ }^{4}$ Donovan-Memorandum vom 10.6. 1941, abgedruckt in Troy, Donovan, S. $419 \mathrm{f}$.
} 
ren Studien und Analysen abhelfen. Der Mitarbeiterstab dieser Abteilung umfaßte in der Aufbauphase zwar kaum mehr als ein Dutzend Fachleute, doch zeichneten sich diese durch große Kompetenz und hohe Einsatzbereitschaft aus ${ }^{5}$. Der renommierte Wirtschaftswissenschaftler Edward S. Mason, der als Mitglied des Board of Analysts die Leitung der Economics Division übernommen hatte, rekrutierte seine ersten Mitarbeiter aus den Rängen der besten Harvard- und Yale-Absolventen, darunter Emile Despres vom Federal Reserve Board ${ }^{6}$, der als außergewöhnlich befähigt und überaus ehrgeizig charakterisiert worden ist, sowie Wilfred Malenbaum, der als einer der besten Nachwuchskräfte unter den Agrarökonomen galt'. Auch Calvin Hoover, der sich in den dreißiger Jahren als Experte für die sowjetische und deutsche Wirtschaft profiliert hatte ${ }^{8}$, gehörte 1941 zum Mitarbeiterstab der Economics Division.

Bereits im Dezember 1941 stellten die R\&A-Wirtschaftswissenschaftler eine umfassende Studie zur "German Economic and Military Position“ fertig", die als grundlegend für die gesamte weitere Arbeit der Abteilung in diesem Bereich gelten kann und als erste ausführliche R\&A-Studie auch dem Präsidenten vorgelegt wurde ${ }^{10}$. Die mehr als 200 Seiten starke Studie setzt sich aus zwei Teilen zusammen, deren erster eine Sammlung von sieben Analysen der wichtigsten wirtschaftlichen Faktoren beinhaltet, während der zweite Teil eine Zusammenfassung der Ergebnisse dieser Analysen und die daraus zu ziehenden Schlußfolgerungen enthält.

Schon allein in ihrer formalen Gestaltung war diese Arbeit vorbildlich, denn mit Blick auf die chronisch überladenen Schreibtische der Washingtoner Entscheidungsträger war jeder Einzelstudie eine ein- bis zweiseitige Zusammenfassung der wichtigsten Ergebnisse vorangestellt, während der Haupttext selbst das jeweilige Thema ausführlich - und wissenschaftlich ordentlich mit Fußnoten belegt - abhandelte. Dieser formale Aufbau wurde für alle umfangreicheren R\&A-Studien übernommen, was - zumindest in der Theorie - ihren Gebrauchswert beträchtlich erhöhte.

Doch auch hinsichtlich ihres Inhaltes waren die vorgelegten Analysen bemerkenswert. In mehreren wichtigen Punkten kamen die Mitarbeiter der Economics Division nämlich zu Ergebnissen, die nicht nur im Widerspruch zu allgemein vorherrschenden Vorstellungen standen, sondern auch manche Schätzungen des British Ministry of Economic Warfare in Frage stellten - und immerhin galt dieses Ministerium in Washington weithin als ,the highest authority on the enemy economy “11. Davon unbeeindruckt be-

${ }^{5}$ Zur personellen Zusammensetzung der Economics Division siehe auch Katz, Foreign Intelligence, $\mathrm{S} .97 \mathrm{ff}$.

6 Während der OSS War Report I, S.56f. Mason als Leiter der Economics Division nennt, wird in NA, RG 226, R\&A-History, (IV), S.3f., behauptet, daß dieser als Mitglied des Board of Analysts Despres für diesen Posten gewonnen habe; vgl. auch Winks, Cloak, S. 86.

7 NA, RG 226, R\&A-History, (IV), S.3.

${ }^{8}$ Vgl. Hoover, The Economic Life of Soviet Russia, und ders., Germany Enters the Third Reich, sowie ders., Dictators and Democracies. Hoover arbeitete jedoch nur während der Aufbauphase des COI in der Economics Division; später (wahrscheinlich im Frühjahr 1942) wechselte er zur Secret Intelligence Abteilung. Unmittelbar nach dem Krieg fungierte er für einige Monate als wirtschaftspolitischer Berater der amerikanischen Besatzungsbehörden in Deutschland, vgl. dazu Hoover, Memoirs of Capitalism, S. $195 \mathrm{ff}$.

9 NA, RG 59, R\&A 214 A, B, 12.12. 1941.

${ }^{10}$ FDRL, PSF/Subject, OSS, b $163, \mathrm{f} 1$, sowie b $165, \mathrm{f} 7$.

${ }^{11}$ NA, RG 226, R\&A-History, (IV), S. 11; vgl. dazu auch OSS War Report I, S. $56 \mathrm{f}$. 
trachtete man in R\&A manche Voraussagen dieses Ministeriums, die in ihrer für Deutschland düsteren Perspektive dem Wunschdenken der Alliierten entgegenkamen, mit großer Skepsis. So lehnten die Mitarbeiter der Economics Division in ihren eigenen Analysen zur Rohstoffversorgung Deutschlands die vom Ministry of Economic Warfare vertretene Ansicht ab, daß Deutschland im Frühjahr 1942 mit einem Chrommangel konfrontiert sein werde und daß für die zweite Jahreshälfte eine bedenkliche Kupferknappheit zu erwarten sei. Ebensowenig war nach den Berechnungen von R\&A zu erwarten, daß Deutschland, wie manche Schätzungen besagten, seine Rohölbzw. Petroleumvorräte im Kalenderjahr 1942 erschöpfen würde. Auch im Hinblick auf die Transportsituation hielten die R\&A-Wissenschaftler Voraussagen ernsthafter Schwierigkeiten für unangemessen; lediglich für den Rußlandfeldzug sahen sie während des Winters Engpässe für die Deutschen voraus.

Bei ihren Berechnungen zur Entwicklung der personellen Stärke der deutschen Streitkräfte kamen die Mitarbeiter der Economics Division zu dem Schluß, daß aufgrund des Geburtenrückganges zwischen 1914 und 1918 nur 7,2 Millionen Mann im idealen Kampfalter zwischen 21 und 33 Jahren zur Verfügung stünden, so daß Deutschland in einem länger dauernden Krieg nicht in der Lage sein würde, eine ausreichende Zahl von Soldaten zu mobilisieren. Diese Einschätzung stand wiederum in krassem Gegensatz zu der weitverbreiteten Ansicht, daß die Versorgung der deutschen Streitkräfte mit Nachwuchssoldaten zu den wenigen Faktoren gehöre, die die militärische Schlagkraft Deutschlands kaum beeinträchtigen würden ${ }^{12}$.

In ihrer Analyse der „German Manpower Resources“ insgesamt vertraten die Experten der Economics Division die Ansicht, daß die durch den Krieg abgezogenen Arbeitskräfte teilweise durch die verstärkte Beschäftigung von Frauen, die Ausbeutung von Fremdarbeitern sowie eine Erhöhung der Arbeitszeit kompensiert würden. Akkurate und verläßliche Schätzungen der Kriegsgüterproduktion hielt man jedoch für schwierig, denn obwohl man glaubte, ein Absinken der Produktivität feststellen zu können, ließ sich nicht absehen, ob dies eine dauerhafte Erscheinung sei und wann sich daraus entscheidende Engpässe für die deutsche Produktion von kriegswichtigen Gütern ergeben würden.

Im Hinblick auf die Versorgung der deutschen Bevölkerung mit Nahrungsmitteln kamen die Mitarbeiter der Econmics Division zu dem Schluß, daß der Kalorienbedarf vorläufig noch gedeckt werden könne, wenn auch auf lange Sicht ein Vitamin- und Mineralstoffmangel zu erwarten stünde, der sich in einer größeren Infektionsanfälligkeit niederschlagen könnte. Zwar gebe es Versorgungsengpässe für Fleisch, Fette und Öl, doch glaubte man in R\&A, daß sich diese für 1942 noch nicht kritisch auswirken würden ${ }^{13}$. Die von der Economics Division vertretene Ansicht, daß die Nahrungsmittelversorgung in Deutschland insgesamt nicht so angespannt sei, daß sie in absehbarer Zeit den Kriegsverlauf zugunsten der Alliierten beeinflussen würde, war wiederum „directly contrary to the prevailing opinion, which characterized not only the public but

12 NA, RG 226, R\&A-History, (IV), S. $11 \mathrm{f}$.

${ }^{13}$ Vgl. auch NA, RG 59, R\&A 84, "The War and German Nutrition“", (COI, Food and Agriculture Section, Special Memorandum EC 8), 5.12. 1941, sowie NA, RG 59, R\&A 834, „The German Position with Respect to Food and Textiles", Dezember 1941; beide Studien sind offenbar in Vorbereitung der hier besprochenen umfassenden Analyse erstellt worden. 
also high intelligence circles, where memories of a hungry 1918 Germany produced wishful predictions of early starvation and collapse ${ }^{\text {"14 }}$.

Neben diesen Analysen der deutschen Wirtschaft enthält R\&A 214 noch einen Bericht zur „German Morale“. Im Rahmen einer Studie zum wirtschaftlichen und militärischen Potential eines gegnerischen Staates mag ein solcher Bericht zunächst befremdlich erscheinen, zumal dieses Thema kaum zum üblichen Arbeitsbereich von Wirtschaftswissenschaftlern gehörte. Doch im Verständnis von R\&A konnte die „Moral“15 der Bevölkerung eines gegnerischen Staates durchaus als komplexer Wirtschaftsfaktor gesehen werden: Ihre Aufrechterhaltung erforderte eine akzeptable Versorgung mit Nahrungsmitteln, und diese wiederum war ein entscheidender Faktor für die Produktivität der Bevölkerung. Dementsprechend maßen die Nationalsozialisten einer möglichst guten Versorgungslage nach Ansicht der R\&A-Wissenschaftler große Bedeutung bei ${ }^{16}$. Auch in Donovans Verständnis war die „Moral“ von Zivilbevölkerung und Streitkräften ein wichtiges Element des Kampfpotentials eines Gegners und deshalb in einer Analyse zur Gesamtstärke dieses Gegners unverzichtbar. In diesem Ansatz werden die innovativen, wenn auch mitnichten klar durchdachten Aspekte von Donovans Verständnis moderner Nachrichtendienstarbeit sichtbar, die sich vor allem in dem Bestreben niederschlugen, den neuen, schillernden und - wie sich zeigen sollte - so schwer zu definierenden Bereich von „psychological warfare" in den Griff zu bekommen. Die R\&A-Wissenschaftler wiesen im Hinblick auf die „German Morale“ denn auch vor allem auf die Rolle und Bedeutung von Propaganda hin. Sie sahen einerseits die Gefahr, daß die von der nationalsozialistischen Propaganda formulierte Alternative zwischen totalem Krieg und totalem Sieg - oder aber totaler Vernichtung - zu einer erbitterten und auch noch in einer völlig verzweifelten Situation durchgehaltenen Verteidigung führen könne; andererseits sah man aber auch die Chance, daß eine wirksame alliierte Gegenpropaganda den Krieg spürbar verkürzen könne, falls es besser als bislang gelinge, "to put across a message of hope - eine Beurteilung, in der sich schon früh die spätere Ablehnung der Forderung nach „Unconditional Surrender" durch R\&A abzeichnet.

${ }^{14}$ NA, RG 226, R\&A-History, (IV), S.10f. Was die Lebensmittelversorgung in Deutschland betraf, so war die Beurteilung der R\&A-Wirtschaftswissenschaftler tendenziell richtig, auch wenn es zum Teil große regionale Unterschiede gab, worauf in R\&A-Studien immer wieder hingewiesen wird. Erst in Schätzungen, die schon über das Kriegsende hinauswiesen, wurden schließlich massive Versorgungengpässe vorausgesehen, so z.B. in NA, RG 226, R\&A 2773, "Significant Developments in the German Food Position", 9.1. 1945.

15 Der englische Begriff ${ }_{n}$ morale“ ist mit "Stimmung ${ }^{*}$ nur unzureichend zu übersetzen, obwohl R\&A-Studien zur "German Morale“ durchaus versuchten, die "Stimmung" der Bevölkerung zu ergründen. „Stimmung“ wurde dabei allerdings als Funktion einer Vielzahl von Faktoren begriffen; letztlich ging es stets um die Frage, wie deutlich die vielfältigen Auswirkungen des Krieges die „Arbeitsmoral“ der Zivilbevölkerung und die „Kampfmoral“ der Truppen beeinträchtigten bzw. wo die psychologische Kriegführung der Alliierten ansetzen könnte, um aufkeimende Unzufriedenheit, Widerstreben und Ängste in ihrem Sinne zu lenken und zu nutzen. In Anlehnung an die Begriffe "Arbeitsmoral“ und "Kampfmoral" wird der englische Begriff "morale" hier mit dem (stets in Anführungszeichen gesetzten) deutschen "Moral" wiedergegeben.

16 NA, RG 59, R\&A 834, „The German Position with Respect to Food and Textiles“, Dezember 1941; vgl. auch die spätere Serie NA, RG 59, R\&A 2215.1 (-5), „Monthly Review of Economic and Morale Factors Bearing on German Unconditional Surrender“, 24.12. 1943-26.4. 1944. 
Als erste umfassende Deutschlandstudie der Economics Division bildete R\&A 214 die Grundlage für die weitere Arbeit der Abteilung in diesem Bereich. Aufbauend auf diese Studie stand ab Frühjahr 1942 das Bemühen, die Grundstrukturen der wirtschaftlichen Position des Gegners sichtbar zu machen, im Mittelpunkt der Arbeit der Economics Division. Analog zu den in R\&A 214 abgedeckten Themenkomplexen etablierten sich die etwa zwei Dutzend wissenschaftlichen Mitarbeiter der Abteilung nun in den Sektionen Industrial Resources, Military Supplies, Agriculture and Standard of Living sowie Labor Supply ${ }^{17}$. Bereits im März 1942 legten die R\&A-Wirtschaftswissenschaftler eine weitere umfassende Studie zur Wirtschaftslage in Deutschland vor, wobei sie wiederum zu dem Ergebnis kamen, daß keine für den unmittelbaren Kriegsverlauf entscheidenden Engpässe bestünden ${ }^{18}$.

Grundsätzlich wiesen die Experten der Economics Division immer wieder darauf hin, daß der weitere Kriegsverlauf für alle Schätzungen einen beträchtlichen Unsicherheitsfaktor darstelle, da sich nur schwer vorhersagen lasse, inwieweit die deutsche Wirtschaft einerseits durch den Krieg gefordert und eventuell überfordert würde, und inwieweit andererseits Engpässe durch eventuelle deutsche Gewinne kompensiert werden könnten. Obwohl sich die Wirtschaftswissenschaftler dieser Problematik bewußt waren, sollte sie ihnen letztlich doch zum Fallstrick werden, was auch in der R\&AHistory nicht verschwiegen wird: „evaluations of the German economy, although they were [...] at first accurate, were subsequently to a considerable extent distorted by the belief that the German economy had by 1940 or 1941 been mobilized for war to an extent approaching the limits of the economy's capability "19. Spätere Zahlen zeigten, daß diese Annahme nicht gerechtfertigt gewesen war und daß ein weiterer Ausbau der deutschen Wirtschaftskapazität möglich war und auch erfolgte ${ }^{20}$.

Diese fehlerhafte Einschätzung mag durch die wenig sinnvolle interne Struktur der R\&A-Branch begünstigt worden sein ${ }^{21}$. Die anfängliche Trennung zwischen funktionalen und regionalen Abteilungen und die unzureichende Koordination der Arbeit der verschiedenen Abteilungen fielen zwar kaum ins Gewicht, solange man sich in der Economics Division auf die Errechnung und Analyse von Daten beschränkte, die weitgehend unabhängig von politischen oder soziologischen Faktoren gesehen werden konnten. Umfassendere Studien wie die Schätzungen zur gesamtwirtschaftlichen Situa-

17 NA, RG 226, R\&A-History, (IV), S. 8; dieser Aufbau der Economics Division wurde im wesentlichen beibehalten, vgl. dazu R\&A Administrative Regulation Number 1, vom 21.1. 1943, in NA, RG 226, E 1, b 2, f: Administrative Office.

18 NA, RG 59, R\&A 386, „Greater Germany, Economic Estimate“, 15.3. 1942, sowie NA, RG 59, R\&A 682, „An Estimate of the Population of Greater Germany by Consumer Groups“, 21.10. 1942.

19 NA, RG 226, R\&A-History, (IV), S. 5. Daß R\&A mit dieser Fehleinschätzung nicht alleine stand, illustriert z. B. ein FEA-Bericht in NA, RG 226, 64678, Foreign Economic Administration, Special Areas Branch, European Enemy Division: „Policies and Control of German Industrial Operations, 1942-1943“, 8.2. 1944.

20 In vielen Bereichen der Rüstungproduktion wurde der Höchststand im Sommer 1944 erreicht, vgl. dazu z. B. Herbst, Der Totale Krieg, besonders S. 253 ff. (mit umfangreichem Literaturverzeichnis); sowie für die von Speer erreichten Wachstumsraten Zilbert, Albert Speer, dort auch zu den Schwierigkeiten, mit denen britische und amerikanische Wirtschaftsexperten bei ihren Schätzungen zur deutschen Wirtschaftskapazität konfrontiert waren, vgl. besonders S. 41 .

${ }^{21}$ NA, RG 226, R\&A-History, (II), S. 14. 
tion des nationalsozialistischen Deutschlands erforderten jedoch die Einbeziehung einer Vielzahl politischer und sozialer Faktoren und damit Kenntnisse, über die die R\&A-Ökonomen bei aller Brillanz nicht immer verfügten. Darüber hinaus erforderten solche Studien aber auch Methoden, die die Mitarbeiter der Economics Division anfangs mit einer gewissen Arroganz gegenüber ihren Kollegen von den „humanities“ schlichtweg ablehnten ${ }^{22}$. Dennoch scheint es hin und wieder zur Zusammenarbeit gekommen zu sein. In einem Bericht über Maßnahmen zur Stärkung der deutschen Kriegswirtschaft ${ }^{23}$ werden als verantwortliche Abteilungen ausdrücklich die „Economics Division in Cooperation With Central European Section" vermerkt. Allerdings gingen die R\&A-Wissenschaftler auch in dieser Studie wieder davon aus, daß die deutsche Wirtschaft schon 1940/41 bis an die Grenzen ihrer Kapazität ausgebaut worden sei. Dies beeinflußte unvermeidlich die Beurteilung der auf eine Erhöhung der Produktion abzielenden Maßnahmen und führte zu dem Fehlschluß: „Miscarriage of plans in Russia and entry of the United States into the war have necessitated squeezing the last measure of output from available productive resources. ${ }^{24}$ Allerdings wurde einschränkend hinzugefügt, daß es keinen Grund gebe anzunehmen, "that the Nazi economy must soon collapse, or even that the scale of supreme effort will soon be reduced“. Partei, Militär und Industrie als wichtigste Machtträger arbeiteten reibungslos zusammen ${ }^{25}$, und, so wurde festgestellt: „A sense of determination, rather than desperation, pervades the new economic measures."

Doch abgesehen von solchen, offenbar seltenen "Gemeinschaftsprojekten“, in denen politische Maßnahmen zur Leitung der Kriegswirtschaft berücksichtigt wurden, spezialisierten sich die R\&A-Wirtschaftsexperten früh auf komplexe Kapazitäts- und Bedarfsberechnungen, zu denen Geistes- und Sozialwissenschaftler nicht viel beitragen konnten. Die Frage nach etwaigen Engpässen in der Rohstoffversorgung sowie der Produktion kriegswichtiger Güter Deutschlands und der Achse nahm bei diesen Analysen eine zentrale Stellung ein. Die Kenntnis solcher Engpässe versprach zum einen die Möglichkeit, Rückschlüsse auf den wahrscheinlichen Kriegsverlauf zu ziehen, zum anderen konnte sie wertvolle Anhaltspunkte für die Planung einer alliierten Strategie bieten, die auf eine Verschärfung dieser Engpässe abzielte. Besonders die unter Leitung des aus Deutschland emigrierten Erdölexperten Walter Levy ${ }^{26}$ erstellten Statistiken und Analysen zum Erdölbedarf und -verbrauch Deutschlands und seiner Satellitenstaaten trugen später viel zur Reputation der R\&A-Economics Division bei, die schließlich durch Levy auch in der Enemy Oil Commission der JCS vertreten sein sollte. Der Arbeitsaufwand, den die Sammlung der für derartige Analysen notwendigen Daten erforderte, ist allein schon formidabel: Einigermaßen zugängliche Statistiken

${ }_{22}$ NA, RG 226, R\&A-History, (IV), S. 20; vgl. auch Katz, Foreign Intelligence, S. $97 \mathrm{ff}$.

${ }^{23}$ NA, RG 59, R\&A 658, "The Strengthening of German War Economy", 28.7. 1942.

${ }^{24}$ Vgl. dazu auch NA, RG 59, R\&A 930, „Estimate of the Effect on the German Labor Force of Mobilization Decrees of Early 1943“, 16.6. 1943.

${ }^{25}$ Vgl. dazu auch NA, RG 59, R\&A 379, „Military and Civilian Controls in German War Economy", 4.12. 1942: "There is no overall supreme economic authority in Germany at the present moment. Decisions are reached by agreement between the major power groups which, in the order of importance, are: the Party, Industry, the Armed Forces, and the High Civil Service."

${ }^{26} \mathrm{Zu}$ Levys beeindruckender Karriere in den USA vgl. Biographisches Handbuch der deutschsprachigen Emigration, Bd. I, s.v. Levy, Walter. 
reichten zumeist nur bis 1939, die entsprechenden Zahlen für die vierziger Jahre mußten meist mühselig hochgerechnet werden ${ }^{27}$, und heutzutage ist kaum vorstellbar, wie all dies ohne den Einsatz von Computern überhaupt möglich war.

Ähnliche Analysen wie für den Erdölbedarf wurden auch für andere kriegswichtige Rohstoffe und Industrien wie etwa die deutsche Stahl-, Gummi-, Textil- und Flugzeugindustrie erstell ${ }^{28}$. Immer wieder gelang es den R\&A-Ökonomen dabei mit erstaunlicher Findigkeit, ihr nur dürftiges Datenmaterial auf ungewöhnliche Weise aufzubessern: So verschafften sie sich beispielsweise aus den Seriennummern erbeuteter Maschinenteile Aufschlüsse darüber, welche Fabrik welche Güter in welchem Umfang wann produzierte ${ }^{29}$. Wie R\&A-Chef Langer rückblickend nicht ohne Stolz feststellte, erschloß R\&A damit „military intelligence of the highest order, attained by altogether new and ingenious methods ${ }^{\text {“ } 30}$. Die R\&A-Wirtschaftswissenschaftler waren sich dessen wohl bewußt - „modesty, false or otherwise, was never a failing of the economics division of OSS“, wie einer von ihnen einmal bemerkte ${ }^{31}-$, und gelegentlich machten sie sich sogar die Mühe nachzuweisen, daß Vergleiche ihrer eigenen Arbeit mit britischen Schätzungen eine klare Überlegenheit für die R\&A-Methoden ergaben ${ }^{32}$.

Die ausgeklügelte Identifizierung von Engpässen in der deutschen Wirtschaft sowie Studien über die Auswirkungen von Bombenangriffen versetzten die R\&A-Wissenschaftler schließlich in die Lage, die Voraussetzungen für das von amerikanischen Strategen befürwortete gezielte Bombardieren individueller, militärisch wichtiger Objekte zu schaffen. Das von der Royal Air Force praktizierte Flächenbombardement war nämlich im Urteil der R\&A-Wissenschaftler nicht allzu effizient. So untersuchte R\&A Ende Juli 1943 beispielsweise die Auswirkungen der Luftangriffe auf das Ruhrgebiet $^{33}$ und kam zu dem Schluß, daß zwar beträchtliche Schäden angerichtet worden seien, daß diese aber nicht ausreichten, um den unmittelbaren Kriegsverlauf zugunsten der Alliierten zu beeinflussen, da kein genügend starker Produktionsrückgang erzwungen worden wäre. Auch unter der Annahme, daß britische Luftangriffe wie bislang fortgesetzt werden könnten, seien keine wesentlich besseren Ergebnisse zu erwarten ${ }^{34}$. Gegenüber der britischen Strategie befürwortete R\&A das gezielte Bombardieren kriegswichtiger Objekte mit dem Argument, daß von dieser Strategie sehr viel durch-

${ }^{27}$ Dazu OSS War Report I, S.177; vgl. zur Arbeitsweise der Economics Division auch Katz, Foreign Intelligence, S. $105 \mathrm{ff}$.

${ }^{28}$ Vgl. dazu auch OSS War Report I, S.177.

29 Vgl. auch NA, RG 59, R\&A 883, „Digest of Applications for Foreign Labor From German Industrial Firms", 1.4. 1943; aus Anträgen deutscher Industrieunternehmen auf Zuteilung von fachlich qualifizierten Fremdarbeitern schloß R\&A auf die Produktionsschwerpunkte dieser Unternehmen.

${ }^{30}$ Langer, Ivory Tower, S. 191.

${ }^{31}$ Sidney Alexander, zit. nach Katz, Foreign Intelligence, S. 109.

32 Z.B. NA, RG 59, R\&A 906, „Comparison of American and British Estimates of German Aircraft Production“, 27.5. 1943; NA, RG 59, R\&A 943, „MEW's Estimates of Foreign Workers in Germany: A Case in the Bits and Pieces Method", 20.5. 1943; vgl. in diesem Zusammenhang auch Hinsley, British Intelligence in the Second World War, Bd. II, S. $129 \mathrm{ff}$.

33 NA, RG 59, R\&A 1005, „The Effect of Aerial Bombardment of the Ruhr on Axis War Potential", 29.7. 1943.

34 Vgl. dazu auch NA, RG 59, R\&A 1273, „German Losses in the Hamburg Raids, 24 July - 2 August 1943“, 18.9. 1943. 
schlagendere Erfolge zu erwarten seien ${ }^{35}$. Damit deckte sich die von R\&A vertretene Position mit den grundlegenden Vorstellungen der US-Strategen. Die Umsetzung dieser Strategie war allerdings bislang daran gescheitert, daß es zum einen an den notwendigen theoretischen Kenntnissen über Schwachstellen der feindlichen Wirtschaftsstruktur gefehlt hatte und daß es zudem überaus diffizil war, die entsprechenden Bombardierungsziele zu identifizieren und präzise zu lokalisieren. Beiden Problemen wandte sich im Herbst 1942 eine Gruppe von R\&A-Experten zu - darunter Chandler Morse, Walt W. Rostow, Carl Kaysen und Charles Kindleberger -, die sich in der Londoner US-Botschaft als Enemy Objectives Unit konstituierten. Obwohl die von dieser Einheit geleistete Arbeit die Voraussetzungen für gezielte Bombenangriffe auf strategisch wichtige Objekte schuf ${ }^{36}$, hielten die Briten, zum Teil aus technischen Gründen, an Flächenbombardierungen fest. Nach Kriegsende durchgeführte Studien und Analysen sollten jedoch erweisen, daß die von R\&A vertretene Position vollauf berechtigt gewesen war $^{37}$.

Während die in der Economics Division versammelten Experten so von Anfang an bemerkenswerte Leistungen erbrachten und ihre Arbeit insgesamt sehr wohl geeignet war, die von Donovan als so bedenklich bezeichnete Informationslücke der Washingtoner Entscheidungsträger zu schließen, fand sie anfangs doch kaum Beachtung. Abgesehen vom Präsidenten verfügte die R\&A-Branch in der Aufbauphase schließlich nicht über einen festen Abnehmerkreis, sondern mußte erst für sich und ihre Arbeit „werben", und obwohl gerade für die Art von Studien, wie sie die Economics Division ausarbeitete, beträchtlicher Bedarf bestand, galt R\&A im riesigen Apparat der Washingtoner Bürokratie zunächst doch nur als eine weitere obskure Neuerung. So wurde etwa eine von R\&A Anfang 1942 ausgearbeitete Studie über die Kapazität deutscher Nachschublinien für die Front in der Sowjetunion nicht etwa auf Anfrage des War Department oder der Joint Chiefs of Staff, sondern auf abteilungsinterne Initiative hin erstellt, „because it seemed to the staff of the division to be a critical question which required answer ${ }^{\alpha 38}$. Aufgrund des dürftigen Datenmaterials, das für die Beantwortung dieser „kritischen Frage“ zur Verfügung stand, war die Arbeit an diesem Projekt schon schwierig genug; die Beschaffung des notwendigen Papiers für die Vervielfältigung der mühsam erarbeiteten Ergebnisse sollte sich kaum weniger problematisch gestalten, und als der Bericht schließlich vorlag, mußte R\&A damit hausieren, „[until it]

35 Siehe dazu OSS War Report I, S.177f.; Corson, Armies of Ignorance, S. 169f.; zum Folgenden vor allem Katz, Foreign Intelligence, S. $112 \mathrm{ff}$.

$36 \mathrm{Vgl}$. auch die Arbeit des britisch-amerikanischen Combined Intelligence Objectives Subcommittee in NA, RG 226, E 1, b 7.

37 Vgl. dazu Cooke/Nesbit, Target Hitler's Oil, S. 108 ff., sowie NA, RG 59, R\&A 2768, „Outline of a Post-Hostilities Study of the Political, Administrative and Morale Effects of Air Raids in Germany", 12.12. 1944; ein entsprechendes Projekt war im November 1944 mit der Einsetzung des United States Strategic Bombing Survey initiiert worden; diese Materialien befinden sich in NA, RG 243, vgl. Daniels (Hrsg.), Guide to the Reports of the United States Strategic Bombing Survey; MacIsaac, Strategic Bombing in World War Two.

38 NA, RG 226, R\&A-History, (IV), S.6ff.; bei der angesprochenen Studie handelt es sich um NA, RG 59, R\&A 217 A, "The German Supply Problem on the Eastern Front June 22 - December 6, 1942" [sic!], 25.3. 1942. In NA, RG 226, R\&A-History, (II), S.6, wird diese Studie als Beispiel für die erstmalige Anwendung der deduktiven Methode zitiert, ebenso in OSS War Report I, S. 58; vgl. dazu auch Katz, Foreign Intelligence, S. $105 \mathrm{ff}$. 
won its way slowly to the attention of those who should have been concerned from the first“.

Nachdem der COI im Juni 1942 als Office of Strategic Services den JCS unterstellt wurde, änderte sich dies allmählich, und besonders die hochspezialisierten Studien der R\&A-Wirtschaftswissenschaftler stießen auf zunehmendes Interesse. Ein Rechenschaftsbericht der Abteilung für das Jahr 1943 listet schließlich eine ganze Reihe von Punkten auf, in denen die R\&A Economics Division den von ihr vertretenen Positionen Gehör und Geltung verschaffen konnte ${ }^{39}$. Wie die Mitarbeiter der Abteilung dabei nicht ohne Befriedigung vermerkten, hatten sich ihre Berechnungen zur Lebensmittel- und Rohstoffversorgung Deutschlands gegenüber den ursprünglich akzeptierten - für die Alliierten optimistischeren - Schätzungen letztlich durchgesetzt. Die R\&A-Wirtschaftswissenschaftler beanspruchten überdies, durch die Entwicklung neuer Methoden zuverlässigere Berechnungen der den Deutschen zur Verfügung stehenden Flugzeuge, Panzer, Lastfahrzeuge und Gewehre ermöglicht zu haben. Einen weiteren wichtigen Beitrag glaubte man durch die detaillierten Studien über die Effizienz von Flächenbombardierungen geleistet zu haben. Besonders stolz war man in R\&A aber offensichtlich, daß die von der Abteilung vorgelegten Zahlen über Verluste der deutschen Streitkräfte von britischen Stellen - „despite strong initial opposition“ letztlich akzeptiert worden waren.

Diese "more striking accomplishments" waren nicht zuletzt deshalb möglich, weil die Economics Division die Experimentierphase, durch die die meisten anderen R\&A-Abteilungen gingen, weitgehend vermeiden konnte. Wenn der OSS War Report darauf verweist, daß auch in der an sich problematischen Aufbauphase von R\&A "work of definite value" geleistet worden $\operatorname{sei}^{40}$, so ist dabei in erster Linie an die Arbeit der R\&A-Wirtschaftsexperten zu denken. Bereits mit ihrer ersten umfassenden Studie war es ihnen gelungen, Themen und Fragestellungen zu formulieren, die eine solide Basis für ihre weitere Arbeit legten, wobei ihnen freilich zugute kam, daß ihre Aufgaben von Anfang an sehr viel klarer definiert waren als die ihrer Kollegen, die einen Beitrag zur psychologischen Kriegführung leisten sollten. Der Arbeitsbereich der Economics Division war dagegen eher konventionell und galt zudem von vornherein als überaus wichtig. Das aus abteilungsinternen Papieren immer wieder erkennbare Selbstbewußtsein der R\&A-Wirtschaftswissenschaftler wurde insofern begünstigt und erlaubte es ihnen wiederum, zuweilen gewagte Positionen zu vertreten, die sich letztlich zwar als korrekt erweisen mochten, aber nicht selten mehr auf „inspired guesswork" denn auf konventionellen mathematischen und statistischen Berechnungen beruhten ${ }^{41}$. Insgesamt hatten die R\&A-Wirtschaftswissenschaftler trotz aller auch für sie entstehender Schwierigkeiten einen leichteren Stand als ihre Kollegen von den Geisteswissenschaften, deren „Existenzberechtigung“ im Rahmen eines Geheimdienstes sehr viel umstrittener war.

39 NA, RG 226, E 1, b 3, Accomplishments of the Research and Analysis Branch, OSS, from 1 January 1943 to 28 March 1944 (Europe-Africa Division, Economic Subdivision).

${ }^{40}$ OSS War Report I, S.61.

41 Katz, Foreign Intelligence, S. 109. 


\section{Versuche zur Psychologie des nationalsozialistischen Deutschlands}

Daß ein totalitärer Staat im totalen Krieg mehr als Industrie und Armee mobilisierte, hatte Donovan frühzeitig erkannt. Wie viele Zeitgenossen überschätzte er allerdings die „secret armies“ einer nationalsozialistischen „Fünften Kolonne“, deren subversiven Aktivitäten Zeitgenossen die so unbegreiflich erscheinenden Erfolge der Hitlerschen „Blitzkriege“ wenigstens teilweise zuzuschreiben geneigt waren ${ }^{42}$. Während die „Fünfte Kolonne“ mehr Schimäre als Realität war, reflektierte dieser Begriff doch das Bewußtsein einer neuen Dimension der Kriegführung, die schließlich unter der Bezeichnung "psychological warfare“ ein umstrittener Bestandteil alliierter Strategie werden sollte. Donovan betrachtete "psychological warfare“ als ein neues und faszinierendes, weil potentiell strategisch gewichtiges Element moderner Kriegführung und war offenbar entschlossen, es in den USA "geheimdienstfähig“ zu machen. Dabei hatte aber auch er nur vage Vorstellungen, wie die von ihm immer wieder geforderte "psychological attack against the moral and spiritual defenses of a nation" im einzelnen auszuführen sei. Einer wirkungsvollen Propaganda, deren Ziel es war, die „Moral“ des Gegners zu brechen, kam nach Donovans Verständnis in diesem Zusammenhang jedoch auf alle Fälle beträchtliche Bedeutung zu.

In der Anfangsphase des COI wirkte sich Donovans Enthusiasmus für psychologische Kriegführung und das von ihm in diesem Zusammenhang als so wichtig erachtete „Schlachtfeld“ Propaganda in einem raschen Aufbau der für diese Bereiche zuständigen Abteilungen aus. So nahm der für die Ausarbeitung und Verbreitung von Propaganda zuständige Foreign Information Service (FIS) unter der Leitung von Robert Sherwood im August 1941 als erste COI-Abteilung seine Arbeit auf, wobei die Beobachtung und Aufzeichnung der nationalsozialistischen Propaganda im Herbst und Winter 1941 zunächst den Schwerpunkt der Arbeit darstellte ${ }^{43}$.

Da der COI unmittelbar dem Präsidenten unterstand, wurden ihm die ersten Ergebnisse dieser Arbeit umgehend zugeleitet. Manches, was Donovan anfangs für wert befand, Roosevelt vorgelegt zu werden, wirkt im Rückblick allerdings eher etwas absonderlich und läßt leicht den Verdacht aufkommen, daß eben noch nicht allzuviel von Substanz vorhanden war. So informierte Donovan den Präsidenten am 30. Dezember 1941 beispielsweise: „The Axis radio called President Roosevelt last night, ,That Klinkenputzer in the White House'. For your information, a Klinkenputzer is a scullion." ${ }^{44}$

Im übrigen erhielt Roosevelt wöchentliche Berichte zur "German home propagan$\mathrm{da}{ }^{* 45}$, die vor allem einen Überblick über Themen nationalsozialistischer Radiopro-

42 Vgl. z. B. zeitgenössische Titel wie Spivak, Secret Armies; Carlson [Arthur Derounian], Under Cover. Smith, Shadow Warriors, S. $11 \mathrm{ff}$. dürfte allerdings wiederum die zeitgenössische Überschätzung der „Fünften Kolonne“ überzeichnen, wenn er meint, daß sie die entscheidende Motivation für die Einrichtung der britischen Special Operations Executive gewesen sei; vgl. dazu die Darstellung bei Stafford, Britain and the European Resistance, S.2ff. Als Darstellung zur „Fünften Kolonne“ siehe de Jong, Die deutsche Fünfte Kolonne.

43 Donovan, Memorandum for the President, No. 334, 16.3. 1942, FDRL, PSF/Subject, b 165, f 7.

${ }^{44}$ FDRL, PSF/Subject, OSS, b 163.

${ }^{45}$ Siche z.B. FDRL, PSF/Subject, COI, b 141 (Summary of Axis Broadcasts, 1941), oder PSF/ Subject, OSS, b 165 (Weekly Review of German Home Propaganda). 
gramme gaben, aber auch andere Materialien auswerteten. Die aufmerksame Beobachtung der nationalsozialistischen Propaganda sollte in erster Linie der Entwicklung einer wirkungsvollen alliierten Gegenpropaganda dienen. Sherwoods Stab bewies dabei zuweilen durchaus Humor. Als beispielsweise in einer für die USA bestimmten NaziPropagandasendung versucht wurde, die Amerikaner über die „falschen“ Berichte von Lebensmittelknappheit in Deutschland mit munterem Geplauder über abendliche Cocktailparties von Nazifunktionären „aufzuklären“, wurde die Sendung einfach übersetzt und auf Kurzwelle fürs deutsche Publikum übertragen. „The German people, getting along on ersatz beer and black bread, listened“, so meldeten zumindest amerikanische Zeitungen und notierten mit Befriedigung über diesen „U.S. Coup“, $\mathrm{daß}$ die Nazis diese Sendung seitdem aus dem Programm genommen hatten ${ }^{46}$.

Hin und wieder versorgte Donovan auch Roosevelt selbst mit Material für Gegenpropaganda. So übermittelte er dem Präsidenten am 18. Dezember 1941 beispielsweise eine Übersetzung des Fuldaer Hirtenbriefes vom 26.Juni 1941 - „the first complete text that could be obtained“ - und vermerkte: „I thought that you might want it as possible source material in future speeches ${ }^{\text {"47}}$; und am 6. Januar 1942 sandte er dem Präsidenten einen Auszug aus einer Predigt des Bischofs von Münster vom 20.Juli 1941, in der der Bischof Gestapo-Aktionen gegen Kirchenbesitz und Orden kritisiert hatte nach Ansicht des Katholiken Donovan wiederum „a useful source for propaganda"48.

Man kann nicht unbedingt davon ausgehen, daß Roosevelt keinen Sinn für solche zuweilen eher kuriose Einzelmeldungen hatte. Mit Interesse scheint er beispielsweise ein ihm von Donovan am 19. Dezember 1941 zugesandtes Memorandum gelesen zu haben, in dem der britische Historiker Wheeler-Bennett ausführte, daß der deutsche Generalstab bald versuchen würde, den Westmächten einen Frieden anzubieten. Durch Tauschungsmanöver sollten Großbritannien und die USA glauben gemacht werden, daß die deutschen Militärs die Nazis entmachtet hätten und nun zusammen mit einigen angeblich gemäßigten Zivilisten - wie Hjalmar Schacht, „beloved of big banking the world over“, und Graf Schwerin von Krosigk, „a former Rhodes Scholar and so gentlemanly and civilized“ - bereit seien, Zugeständnisse für eine Friedensregelung zu machen. Roosevelt notierte daraufhin an Donovan am 22. Dezember 1941: „I think that this is so plausible and yet such an insult to intelligence that it should be broadcast throughout this country and England and South America by some person not connected with the Administration. "49

Wollte man Psychologie und Propaganda aber tatsächlich als wirksame Waffe im modernen, totalen Krieg einsetzen, so konnten derartige ad-hoc-Attacken längerfristig kaum genügen. Donovan selbst hatte in seinem Memorandum vom 10.Juni 1941 darauf hingewiesen, daß der von den Deutschen so erfolgreich praktizierte Einsatz des Radios noch perfektioniert werden könne, daß dafür aber Planungen notwendig seien, die sich auf verläßliche und korrekte Information gründen müßten. Neben der Beobachtung

${ }^{46}$ FDRL, PSF/Subject, b 165, f 8, Donovan, Memorandum for the President, No. 358, 27.3. 1942.

47 FDRL, PSF/Subject, OSS, b 163, $f 2$.

48 FDRL, PSF/Subject, OSS, b 163, 6.1.1942.

49 FDRL, PSF/Subject, OSS, b 163. Zur Tätigkeit Wheeler-Bennetts während des Krieges siehe Kettenacker, Der nationalkonservative Widerstand aus angelsächsischer Sicht, in: Schmädeke/ Steinbach (Hrsg.), Der Widerstand gegen den Nationalsozialismus, S. $722 \mathrm{f}$. 
der nationalsozialistischen Propaganda kam dem Sammeln von Informationen über die "Moral" der deutschen Bevölkerung in diesem Zusammenhang ein hoher Stellenwert zu, denn eventuelle Schwachstellen in der deutschen „Moral“ konnten schließlich Ansatzpunkte für die alliierte Gegenpropaganda bieten.

Der britische Nachrichtendienst hatte bereits eine aufwendige Untersuchung über die Stimmung der deutschen Bevölkerung begonnen: Von Frühjahr bis Herbst 1941 hatte die Imperial Censorship in Bermuda Zehntausende von Briefen aus Deutschland abgefangen und ausgewertet „[in] an attempt to provide a kind of everyday history of Germany during the last six months ${ }^{\text {" }}{ }^{50}$. Die daraus gewonnenen Erkenntnisse, die auch dem COI zur Verfügung gestellt wurden, dürften den enormen Arbeitsaufwand allerdings kaum gelohnt haben, denn zusammenfassend ließ sich offenbar lediglich feststellen, daß in der deutschen Bevölkerung zwar Trauer und Bedrückung wegen der vielen Toten an der Ostfront herrsche, daß aber die "Moral“ insgesamt durchaus nicht schlecht sei. Selbst dieses vage Bild mußte noch durch den Hinweis auf eventuelle Verzerrungen durch die deutsche Zensur bzw. die Angst vor ihr relativiert werden. Der Versuch, die künftige Entwicklung der „Moral“ der deutschen Bevölkerung zu skizzieren, mißlang den britischen "Zensoren" schließlich vollständig, da die Bandbreite der Manipulationsmöglichkeiten eines totalitären Regimes in ihrer Beurteilung völlig außer acht blieb: „One major setback, or even prolonged failure to end the present slaughter, and the German will to sacrifice and conquer might hang dangerously in the balance."

Der im Aufbau befindliche COI konnte mit derlei umfassenden, wenn auch im Ergebnis kaum befriedigenden Untersuchungen zunächst nicht konkurrieren. Seine Tätigkeit auf diesem Gebiet beschränkte sich anfänglich meist auf die Sammlung von Informationen zur deutschen „Moral“. Wichtig war in diesem Zusammenhang die Arbeit der unter Leitung des mit Donovan befreundeten Geschäftsmannes und ehemaligen Kriegsberichterstatters G. Edward Buxton stehenden Oral Intelligence Unit (OI $)^{\text {s. }}$. Hauptaufgabe ihres kleinen, offenbar aber hochqualifizierten Mitarbeiterstabes war die Befragung von in New York ankommenden Flüchtlingen aus Europa. OI arbeitete dafür eng mit dem Büro des British Security Office in New York zusammen und nahm darüber hinaus Kontakte zu den Nachrichtendienststellen einiger von Deutschland besetzter europäischer Staaten sowie zu Widerstandsorganisationen auf. Diese Kontakte erlaubten es OI-Mitarbeitern, nicht nur Kurzinterviews mit gerade ankommenden Flüchtlingen durchzuführen, sondern auch längerfristig vorbereitete, intensivere Befragungen vorzunehmen. $\mathrm{Da}$ aktuelle und unzensierte Nachrichten über die Situation im besetzten Europa zunehmend schwieriger zu erhalten waren, stellten diese Interviews eine wertvolle Informationsquelle dar; nicht zuletzt erhoffte man sich von ihnen die minutiösen Detailinformationen zum nationalsozialistischen Alltag, die für die Vorbereitung von Agenten auf einen eventuellen Einsatz in Deutschland oder in deutsch besetzten Gebieten unerläßlich waren. Die Mitarbeiter von OI befragten aber nicht nur Flüchtlinge, sondern auch von Europareisen zurückkehrende Vertreter von Banken, Firmen und Wohlfahrtsorganisationen.

50 NA, RG 226, 5187; zur Arbeit der Bermuda Censorship vgl. Hyde, Secret Intelligence Agent, S. $57 \mathrm{ff}$. Wie bereits angedeutet, werteten auch amerikanische Stellen abgefangene Briefe aus Deutschland (und anderen Ländern) aus, siehe z. B. die COI-Materialien für 1941/42 im NAIndex zur RG 226, Plain Number Files, $L$ and XL Files, 0.1 .

${ }^{51} \mathrm{Zu}$ Aufgabenkreis und Arbeitsweise der OI-Unit siehe OSS War Report I, S. 11, $61 \mathrm{ff}$. 
Die Arbeit der New Yorker OI-Unit wird im OSS War Report recht hoch bewertet, zumal OI die erste COI-Abteilung war, die selbständig „raw intelligence“ sammelte. Der spürbare Rückgang des in den USA eintreffenden Flüchtlingsstromes sollte im September 1942 allerdings zur Auflösung der OI-Unit führen. Gleichzeitig wurde aber eine R\&A-Außenstelle in New York eingerichtet, die die Arbeit von OI in kleinerem Rahmen weiterführte und R\&A zudem mit Materialien versorgte, die öffentliche und private Organisationen in New York bieten konnten ${ }^{52}$.

Darüber hinaus verfügte der COI seit Dezember 1941 mit der Foreign Nationalities Branch (FN) über eine Abteilung, die - teilweise ebenfalls in enger Zusammenarbeit mit Exilanten - die Aktivitäten ausländischer Staatsangehöriger und ihrer Organisationen beobachtete, um zu analysieren, wie sich die politischen Bedingungen im Ausland in den USA niederschlugen ${ }^{53}$. Die Anregung zur Einrichtung dieser COI-Abteilung war vom State Department ausgegangen, das sich zu seinem Unbehagen mit einer wachsenden Anzahl von Exilanten konfrontiert sah, die darum bemüht waren, Kontakte mit Mitarbeitern des Ministeriums zu knüpfen, da sie sich davon Vorteile für sich selbst bzw. die Politik der von ihnen vertretenen Organisationen erhofften. Under Secretary of State Sumner Welles hatte Donovan deshalb vorgeschlagen, innerhalb des COI eine Abteilung einzurichten, die das State Department mit Informationen und Analysen über prominente Exilanten sowie die zahlreichen Exilantenorganisationen versorgen würde. Obwohl sich im State Department auch Widerstände gegen diese enge Zusammenarbeit mit dem neuen COI regten, autorisierte Secretary of State Cordell Hull dieses Arrangement im Januar 1942. Direktor der FN-Branch wurde der auch im Board of Analysts vertretene Diplomat John C. Wiley, der zu diesem Zeitpunkt bereits auf eine reiche Erfahrung an verschiedenen Botschaften in Europa - darunter Paris, Berlin, Kopenhagen und Warschau - verweisen konnte ${ }^{54}$. Wileys Stellvertreter und Assistent war DeWitt C. Poole, ebenfalls ein Diplomat, der wie Wiley an mehreren europäischen Botschaften tätig gewesen war ${ }^{55}$. Neben ihrem regulären Mitarbeiterstab beschäftigte die FN-Branch auch immer wieder Exilanten, und die Kontakte der Abteilung sollten später für die mit der Beschaffung von „secret intelligence“ und der Durchführung von Sabotage- und Guerillaaktionen befaßten COI- bzw. OSS-Abteilungen wichtig werden.

Der Aufgabenkreis der FN-Branch umfaßte zudem die Auswertung der in den Vereinigten Staaten publizierten ausländischen Presse. Während diese Materialien relativ einfach zu beschaffen waren, sahen sich die R\&A-Branch sowie die Abteilungen einiger anderer Regierungsbehörden, für deren Arbeit die Auswertung der nur in Übersee erscheinenden Presse unabdingbar war, bei der Beschaffung dieser Materialien vor immer größere Probleme gestellt. Um dem abzuhelfen, wurde die Einrichtung eines Interdepartmental Committee for the Acquisition of Foreign Publications (IDC) angeregt. Im Dezember 1941 autorisierte Roosevelt die Einrichtung dieses Ausschusses ${ }^{56}$,

52 Ebenda, S. 171.

${ }^{53}$ Ebenda, S.64; zu Einrichtung, Aufgaben und Arbeitsweise der FN-Branch siehe S.15f. und S.63-67; sowie Troy, Donovan, S.109f.; Roosevelts „O.K.“ zur Einrichtung der FN-Branch in FDRL, PSF/Subject, OSS, b 163, f 2, Donovan an Roosevelt, 20.12. 1941.

54 Who's Who in America, 23, S. 2304.

55 Ebenda, S. 1692.

56 FDRL, PSF/Subject, OSS, b 163, f 3, Memo vom 22.12. 1941; vgl. auch OSS War Report I, S.60f., 170; NA, RG 226, R\&A-History, (IDC), S. 1 ff.; zur Arbeit des IDC und seiner ein- 
der daraufhin aus Vertretern von State-, Navy-, War-, Treasury-, Commerce- und Interior Department sowie der Library of Congress, des Office of Facts and Figures, des Board of Economic Warfare und einiger anderer Behörden gebildet wurde. Ursprünglich war das IDC nicht als COI-Abteilung gedacht gewesen, doch Schwierigkeiten bei der Beschaffung und Finanzierung von Zeitungen und Zeitschriften ließen eine Angliederung des Komitees an den COI bzw. OSS schließlich als die praktikabelste Lösung erscheinen. Die Arbeit des IDC wurde für den COI/OSS und besonders für R\&A letztlich sehr wichtig, denn es war oft genug das IDC, das die Zeitungen besorgte, aus denen R\&A wertvolle Daten und Informationen gewann ${ }^{57}$.

Mit der von Donovan versprochenen Analyse und Interpretation der so gesammelten Informationen haperte es jedoch während der ersten Monate des Bestehens des COI zunächst noch unübersehbar. Wie bereits angedeutet, enthielten auch die Berichte und Meldungen, die Donovan an Roosevelt weiterleitete, meist lediglich kaum aufgearbeitete „raw intelligence“. Häufig handelte es sich um relativ kurze und allgemein gehaltene Berichte zur deutschen „Moral“, wobei die einzelnen Meldungen nicht unbedingt ein einheitliches Bild ergaben. So wurden dem Präsidenten Mitte Dezember die Aussagen eines amerikanischen Geschäftsmannes zugeleitet, der nach mehrjähriger Tätigkeit in Berlin Mitte November 1941 in die USA zurückgekehrt war und zu Protokoll gegeben hatte, daß die Lebensbedingungen in Berlin keineswegs schlecht seien, obwohl die Situation von Arbeitern recht schwierig sei. Die „Moral“ müsse als allgemein gut bezeichnet werden; allerdings glaubte der Informant, daß angesichts des Kriegseintritts der USA eine Verschlechterung eintreten würde ${ }^{58}$. Zusammen mit diesem Bericht findet sich in den Akten des Präsidenten ein weiterer Bericht über die "Moral" in Deutschland, in dem zwar behauptet wird, daß die "Stimmung" in letzter Zeit nicht sonderlich gut gewesen sei, gleichzeitig aber betont wird, daß ein starker Glaube an Deutschlands militärische Schlagkraft vorhanden sei, so daß der Kriegseintritt der USA keinen allzu großen Eindruck gemacht habe ${ }^{59}$.

Solange solche Meldungen einfach nur nebeneinandergestellt und kommentarlos weitergereicht wurden, dürfte ihr nachrichtendienstlicher Wert nicht allzu hoch zu veranschlagen sein, und auch als Basis für die Ausarbeitung amerikanischer Propagandaprogramme konnten sie in dieser Form von nur begrenztem Wert sein. Gerade weil Meldungen über die „Moral“ in Deutschland häufig widersprüchliche Informationen enthielten, erforderten sie eine gründliche und kenntnisreiche Analyse bzw. Interpretation. Wurde dies nicht geleistet, so ließ sich aus den beim COI eingehenden Einzelmeldungen jede beliebige Schlußfolgerung ziehen.

So wurde beispielsweise in einem an einen New Yorker Empfänger gerichteten Brief aus Basel die Situation in Deutschland im September 1941 als völlig verzweifelt dargestell $t^{60}$. Ein zehn Seiten umfassender Bericht eines jüdischen Lehrers, der Berlin An-

drucksvollen Leistungsbilanz ebenda, S.71f. (Kilgour an Donovan, 30.4. 1943), sowie Winks, Cloak, S. $101 \mathrm{ff}$.

57 Vgl. z. B. die bei Langer, Ivory Tower, S. 191 f. beschriebene Auswertung von in deutschen Lokalzeitungen erscheinenden 'Todesanzeigen von Gefallenen, aus denen R\&A Aufschlüsse über Truppenstationierungen und deutsche Verluste gewann.

58 FDRL, PSF/Subject, OSS, b 163, f 1, Memo vom 17.12.1941 (William H. Vanderbilt an Donovan).

59 FDRL, PSF/Subject, OSS, b 163 (Donovan Reports, December 12-17, 1941, f 1).

60 NA, RG 226, 4072. 
fang August 1941 verlassen hatte, zeichnete dagegen ein recht differenziertes Bild: Die Versorgungslage sei zwar schwierig, aber durchaus noch in den Grenzen des Erträglichen, andererseits seien Arbeiter durch Überstunden völlig überfordert. Die Gestapo erscheine als „ever-present power", und jeder befände sich in „a hell of fear"61. Ein soeben von einer Europareise zurückgekehrter "Special Agent" konstatierte in seinem Bericht vom 19.September 1941 eine grundlegende Veränderung der Stimmung in Deutschland zwischen Juli 1940 und Juli 1941: Die ursprünglich in der Bevölkerung vorherrschende Siegesgewißheit sei einer gedrückten Stimmung gewichen, und die anfänglich sehr gute Versorgung habe sich massiv verschlechtert ${ }^{62}$. Dagegen beschrieben polnische Agenten im Mai 1942 die wirtschaftliche Lage wieder als akzeptabel und beurteilten die deutsche „Moral“ als relativ gut, obwohl durchaus "signs of serious criticism against the Nazi regime" zu beobachten seien ${ }^{63}$. Ein Schweizer, der drei Semester in Halle studiert hatte, berichtete im August 1942 wiederum: „The people in Germany have the impression of being on the eve of an unavoidable catastrophe." Ihre Kriegsmüdigkeit manifestiere sich in bedrücktem Schweigen, die Lebensmittelversorgung sei schlecht und man sähe viele verwundete und verkrüppelte Soldaten ${ }^{64}$.

Während sich aus diesen Berichten insgesamt als recht eindeutige Tendenz eine Verschlechterung der Versorgungslage und der "Moral“ in Deutschland herauslesen ließ, mochten diejenigen Meldungen, die diesem Bild nicht entsprachen, durchaus verschiedene Strömungen in verschiedenen Gruppen der deutschen Bevölkerung oder auch in verschiedenen Regionen widerspiegeln. Grundsätzlich mußte sich aber die Frage stellen, inwieweit diese Berichte tatsächlich vorhandene Tendenzen wiedergaben oder inwieweit sie vielleicht lediglich die subjektive Wahrnehmung oder Erwartungshaltung des jeweiligen Berichterstatters reflektierten.

Das Bild, das COI-Informationen von der Stimmung in Deutschland zeichneten, stützte sich allerdings bald nicht mehr nur auf diese Art von "raw intelligence“. Ab Frühjahr 1942 legten die Mitarbeiter der Psychology Division eine ganze Reihe von Studien vor, die ein weitaus breiteres Spektrum von Quellen auswerteten. Die Analyse der nationalsozialistischen Propaganda selbst stand dabei im Vordergrund. So wurde beispielsweise in einer im März 1942 fertiggestellten Studie versucht, aus den Themen deutscher Radiosendungen auf Schwachstellen in der „Moral“ der deutschen Bevölkerung zu schließen ${ }^{65}$. In ähnlicher Weise hoffte man, durch die Analyse von mehr als 100 Goebbelsreden und -artikeln Erkenntnisse über „German Domestic Attitudes“ zu gewinnen ${ }^{66}$. Schließlich versuchte R\&A, diejenigen Gruppen der deutschen Bevöl-

${ }^{61}$ NA, RG 226, 509.

${ }^{62}$ NA, RG 226, 5378.

${ }^{63}$ NA, RG 226, 16698.

64 NA, RG 226, 22263.

${ }_{65}$ NA, RG 59, R\&A 616, „The Radio Propaganda Atmosphere of the German Public“, 21.3. 1942; außerdem wurden die Mechanismen der nationalsozialistischen Propaganda analysiert, siehe z.B.: NA, RG 59, R\&A 608, "Design of Propaganda in German Foreign Newsreels", 19.3. 1942, und NA, RG 59, R\&A 701, "Nazi Use of Films in Psychological Warfare“, 22.2. 1943, sowie NA, RG 59, R\&A 634, „Procedures for Developing Morale in the German Armed Forces", 11.5. 1942.

${ }^{66}$ NA, RG 59, R\&A 275, „Goebbels' Statements as Indicators of German Domestic Attitudes“, 15.4. 1942. Die Mitarbeiter der Psychology Division versuchten sich schließlich sogar darin, 
kerung zu identifizieren, die dem nationalsozialistischen Regime kritisch gegenüberstanden und somit als vorrangige Zielgruppen für die alliierte Propaganda in Frage kämen $^{67}$.

Die Ergebnisse dieser Studien und Analysen blieben allerdings meist viel zu vage, um von nennenswertem Nutzen für die Entwicklung einer wirksamen amerikanischen Propaganda zu sein. Man versuchte jedoch auch, aus Befragungen Anhaltspunkte dafür zu gewinnen, welche Art von Propaganda am wirksamsten sei, und offenbar wurden Informanten gelegentlich direkt dazu befragt ${ }^{68}$. So enthielt etwa der bereits zitierte Bericht eines jüdischen Lehrers aus Berlin sowohl Informationen über die Wirksamkeit nationalsozialistischer Propaganda wie Empfehlungen für die Gestaltung alliierter Gegenpropaganda: „Generally speaking, the German people have no war aim that seems attractive enough for them to fight for [...] While the hope for a better future is very small, the fear of a breakdown is very great [...] Only that part of the Nazi propagan$\mathrm{da}$ is effective which exploits the enemy's plans for the partition and destruction of Germany. This part of the propaganda is taken seriously and this fear creates again and again the resolution to go through with it. It is really much more effective than the terror of the Gestapo. Any propaganda from abroad should be related to this knowledge. It should show a way out of the difficulties [... The Treaty of Versailles was a mistake with terrible consequences. It is through it that Hitler has won power. “69

Dieser Bericht, der beim COI am 3. Oktober 1941 einging - und offenbar gelesen wurde, da die Forderung, daß die alliierte Propaganda „a way out of the difficulties“ weisen sollte, unterstrichen und am Rand markiert ist -, könnte als eine Art Grundsatzpapier der R\&A-Branch gelten. Nicht nur die hier hervorgehobene Empfehlung findet sich immer wieder in R\&A-Arbeiten zur alliierten Propaganda - erstmals in der bereits zitierten Analyse der Economics Division zur "German Morale" -, auch die spätere Ablehnung der Forderung nach "Unconditional Surrender" durch R\&A entspricht den hier gegebenen Empfehlungen ebenso wie viele der von der Abteilung in der Kontroverse um die Nachkriegspolitik vertretenen Positionen.

Psychologie konnte nach Donovans Ansicht aber nicht nur bei der Ausarbeitung von Propaganda von Nutzen sein. Mit einem im Frühjahr 1943 initiierten Projekt versuchte Donovan auf ganz andere Weise, die Psychologie in den Dienst der Washingtoner Entscheidungsträger zu stellen. Er beauftragte nämlich William Langers Bruder, den Psychologen Walter Langer, eine umfassende Studie über das „psychological make-up" Adolf Hitlers und die Grundlagen seiner scheinbar magischen Wirkung auf die Deutschen auszuarbeiten ${ }^{70}$. Walter Langer nahm diesen ungewöhnlichen Auftrag an und arbeitete sich in den folgenden Monaten mit einem kleinen Stab von Assistenten durch Berge von Büchern, Zeitschriften und Zeitungsartikeln, die den Patienten

die Psyche des nationalsozialistischen Regimes und seiner Machthaber mit Nutzen für das Militär zu erforschen: NA, RG 59, R\&A 297, „Prediction of German Military Offensives from Speeches of Leaders", 12.6. 1942 - Hauptergebnis der Studie war allerdings, daß kein Propagandamuster erkennbar sei, das Rückschlüsse auf militärische Vorhaben erlaube.

67 NA, RG 59, R\&A 609, "Current German Attitudes and the German War Effort", 19.3. 1942.

${ }^{68}$ Entsprechende Empfehlungen bzw. Beurteilungen der Wirksamkeit alliierter Propaganda finden sich z.B. in NA, RG 226, 6039, 14472, 17180.

69 NA, RG 226, 509.

70 Das Folgende nach Walter Langers Einleitung (S.13ff.) in ders., The Mind of Adolf Hitler. 
auf der Couch ersetzen mußten. Zudem konnte Langer einige ausführliche Gespräche mit Personen führen, die Hitler kannten; so interviewte er zum Beispiel den inzwischen in Montreal lebenden Otto Strasser. Aus dieser Materialsammlung entstand ein mehr als 1000 engbeschriebene Seiten umfassendes „Hitler Source-Book“, das die Basis für Langers im Herbst 1943 auf Drängen Donovans in größter Eile fertiggestellte Analyse Hitlers bildete, die dann an einen geheimgehaltenen, anscheinend sehr kleinen Kreis verteilt wurde.

Zwar ist nicht festzustellen, welche Aufschlüsse und Erkenntnisse die Empfänger dieser Studie aus ihrer Lektüre gewannen, doch immerhin sollte Langer mit manchen seiner Einschätzungen recht behalten. Im letzten Kapitel seiner Studie hatte er beispielsweise die Frage angesprochen, wie sich Hitler wohl in Zukunft verhalten würde. Nach Langers Ansicht war damit zu rechnen, „[that,] as Germany suffers successive defeats Hitler will become more and more neurotic. [...] His public appearances will become less and less." Für das Ende des Krieges und eine unmittelbar bevorstehende deutsche Kapitulation diskutierte Langer acht verschiedene Reaktionen Hitlers und kam zu dem Schluß, daß ein Selbstmord Hitlers in dieser Situation „the most plausible outcome" $\mathrm{sei}^{\mathrm{71}}$.

Insgesamt waren diese Versuche zur Psychologie des nationalsozialistischen Deutschlands und seiner Machthaber wohl zu innovativ, zu unkonventionell, aber auch zu unausgegoren, um im Washington der Kriegsjahre als ernstzunehmender Beitrag zum amerikanischen „war effort“ begrüßt zu werden. Als nicht unproblematisch erwies sich dabei auch, daß Donovan seine manchmal etwas exzentrischen Vorstellungen anfangs allzu deutlich zum Tragen bringen konnte, und die zahlreichen unsystematischen „spot reports", die der OSS War Report als charakteristisch für die anfängliche Arbeit der R\&A-Branch bezeichnet ${ }^{72}$, dürten nicht zuletzt auf Donovans Konto gehen. Sein Ehrgeiz, "psychological warfare“ als eine Art neuer Waffengattung, als „fourth arm“ der amerikanischen Militärmaschinerie zu etablieren, war eher geeignet, Verwirrung zu stiften, solange keine Klarheit über den Begriff selbst, geschweige denn Vorstellungen über Strategien und ihre Umsetzung existierten.

Grundsätzlich muß der Arbeit der mit „psychological warfare“ befaßten R\&A-Abteilungen während des ersten Jahres ihres Bestehens zugute gehalten werden, daß sich R\&A erst im Aufbau befand und daß dabei Pionierarbeit zu leisten war. Fast unvermeidlich gestaltete sich dies als mühsamer „learn-as-you-go “-Prozeß, was selbst im offiziellen OSS War Report durchscheint, wenn vermerkt wird: „The internal structure of the [R\&A] Branch, the focus of its attention, its sources and customers, its techniques and methods of operation, its place within the agency and the Government at large were still to be finally determined as experience was gained. ${ }^{* 73}$ Diejenigen Abteilungen, von denen ein Beitrag zur psychologischen Kriegführung erwartet wurde, mußten sich in diesem Prozeß besonders schwertun, da noch lange unklar blieb, was unter „psychological warfare“ eigentlich genau zu verstehen war.

\footnotetext{
${ }^{71}$ Ebenda, S.213-217; vgl. jedoch Langers Fehleinschätzung der Ereignisse vom 20.Juli 1944 in: Heideking/Mauch (Hrsg.), USA und deutscher Widerstand, S. 101-105.

72 OSS War Report I, S.61.

${ }^{73}$ A.a.O.
} 
